\title{
Dysglycemic Effect and Misuse Prevalence of Fluoroquinolones in ICU Diabetic Patients
}

\section{Rasha Ashmawy ${ }^{1 *}$, Jayda M Ahmed ${ }^{2}$, Ingie A E El Chennawi ${ }^{3}$, George N Anis $^{3}$, Logine A El Shaarawy ${ }^{3}$, Poula Azziz ${ }^{3}$, Aly El-halfawy ${ }^{3}$, Abdelrhman Agamy ${ }^{3}$, Amr Y Bargal ${ }^{3}$, Maged E Ahmed ${ }^{3}$, Nayera $\mathrm{H}$ Fouda $^{3}$ and Ahmed R Farag ${ }^{3}$}

${ }^{1}$ B. Pharm, MSc Biostatistics, Medical Research Institute, Alexandria University, Egypt

${ }^{2}$ Supervisor and Demonstrator at Department of Pharmacy Practice, Faculty of

Pharmacy, Alexandria University, Egypt

${ }^{3}$ Students at Clinical Pharmacy Program, Faculty of Pharmacy, Alexandria University,

Egypt

*Corresponding Author: Rasha Ashmawy, B. Pharm, MSc Biostatistics, Medical

Research Institute, Alexandria University, Egypt.
Received: October 13, 2020

Published: November 18, 2020

(C) All rights are reserved by Rasha

Ashmawy., et al.

\begin{abstract}
Introduction: Quinolones are one of the most favourable antimicrobial class for the treatment of various infectious diseases. Their side effects and toxicity profile have been established years ago. Multiple studies reported dysglycemic events occurring in diabetic patients admitted to the ICU taking fluoroquinolones. The aim of this study is to determine if there is indeed a relation between fluoroquinolones and dysglycemia.

Methods and Materials: A retrospective observational study targeting the ICU patients who were administered levofloxacin, ciprofloxacin, and moxifloxacin. This study was performed during the period from April 2019 to September 2019 (6 months) in 5 different hospitals in Alexandria, Egypt. 350 patients' ICU files were chosen randomly, then a total of 68 diabetic ICU admitted patients receiving broad-spectrum fluoroquinolones for more than 2 days were included in the study.

Results: From 68 patients who recruited in our study, 15 patients take fluoroquinolone with no need and they suffer from change in their blood glucose level without any additional benefit. The most misused fluoroquinolone was Levofloxacin, as $73.3 \%$ of patients with main diagnosis non-infectious treated with Levofloxacin, and $20 \%$ among them suffered from decreased blood glucose level where $13.3 \%$ had significant increase of their blood sugar level.

Conclusion: Dysglycemia caused by fluoroquinolones is an important issue of clinical significance and must be addressed and managed. Dysglycemia occurs by varying degrees and differ between different agents of fluoroquinolones. The effect is more pronounced in diabetics and patients with renal impairment which warrant attention and immediate management. Fluoroquinolone misuse is also another important issue to be addressed as it contributes to increased antimicrobial resistance and exposes the patients to unnecessary side effects some of which might need immediate intervention. Further investigations related to fluoroquinolones prescribing and monitoring is needed to get a better understanding of this existing problem.
\end{abstract}

Keywords: Anti-infective(s); Fluoroquinolones; Toxicity; Dysglycemia; Adverse Events; Misuse; Diabetes

\section{Abbreviations}

ACEIs: Angiotensin Converting Enzyme Inhibitors; AKI: Acute Kidney Injury; B-Blocker: Beta Blocker Drugs; BSL: Blood Sugar
Level; CAP: Community Acquired Pneumonia; COPD: Chronic Obstructive Pulmonary Disease; CNS: Central Nervous System; DVT: Deep Vein Thrombosis; DNA: Deoxyribonucleic Acid; HAP: Hospital 
Acquired Pneumonia; ICU: Intensive Care Unit; VAP: Ventilator Associated Pneumonia; BSL decrease > 30\%: Blood Sugar Level (BSL) Decreases more than 30\% from Initial BSL; BSL increase > 30\%: Blood Sugar Level (BSL) Increases more than 30\% from Initial BSL; BSL $(-30 \%$ to $+30 \%)$ change: Blood sugar level (BSL) Decreases Less than $30 \%$ or Increases Less than 30 from Initial BSL, that could not Harm Patients; Initial BSL: The first random blood sugar level for each patient at hospital admission as routine laboratory investigation and not by capillary blood glucose method to ensure accuracy of results; Final BSL: The second laboratory random glucose level for the same patient after 2 days fluoroquinolone treatment at least; CYP450: Cytochrome P450

\section{Introduction}

Since their discovery 40 years ago, quinolones have been one of the most favourable antimicrobial class for the treatment of various infectious diseases [1-4]. This is because they offer a lot of attributes that made them an ideal class of antibiotics [1-4]. These include their broad spectrum activity, high potency, good bioavailability and availability of both oral and intravenous formulations [2]. Therefore, they are the best choice for physicians to prescribe them in numerous indications, which may cause irrational use of this important class of antibiotics.

The first agent in this class was nalidixic acid which was discovered as a by-product of antimalarial research [1-3]. Quinolone structure represented in figure 1 is not only responsible for its activity but also its side effects profile [1-5]. Over the years, modifications have been made to this basic nucleus to synthesize different numbers of quinolones to improve their properties and overcome antimicrobial resistance [1-5]. Different classes of quinolone antibiotics are represented in figure 2 .

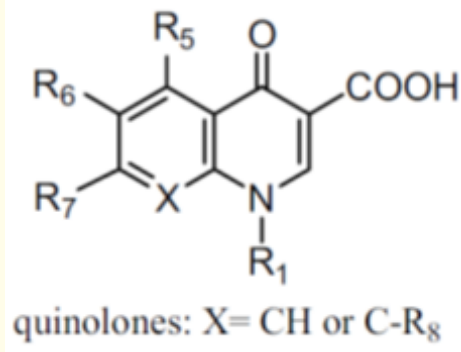

Figure 1: Basic structure of quinolones.

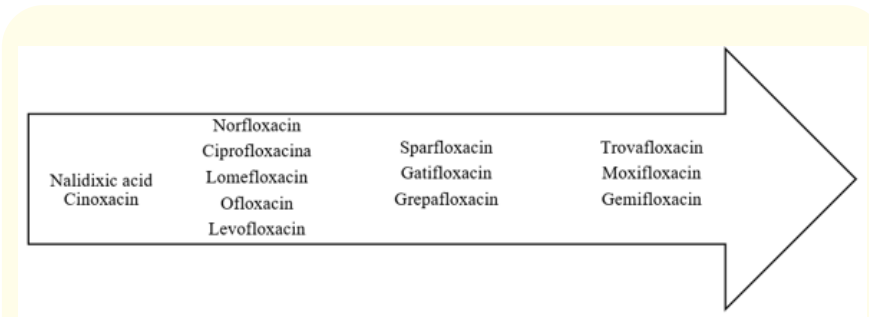

Figure 2: Development of quinolones.

Mechanism of action of quinolones is mainly through inhibition of topoisomerase enzymes (also known as DNA gyrase) specifically topoisomerase II and IV [1-3]. Inhibition of this enzyme prevents DNA replication and thus bacterial multiplication and survival IV [1-3].

Fluoroquinolones are known to have a number of side effects which are unique to this class [4-7]. These side effects range from mild to severe, life threatening side effects class [4-7]. These side effects can be classified according to organ system into cardiovascular, CNS, gastrointestinal, dermatologic, hepatic, renal, musculoskeletal and others $[4,6,7]$. Examples of some of the common side effects include QTc prolongation, nausea/vomiting/ diarrhoea, phototoxicity, renal failure, elevation in hepatic enzymes, hepatitis, seizures and tremors $[4,6,7]$.

Dysglycemia (disturbances in blood glucose levels) have been reported with a number of fluoroquinolones [8]. Both hypoglycaemia and hyperglycaemia have been documented with gatifloxacin which led to label changes [9-12]. Although risk of hypoglycaemia is more common, some reports found that gatifloxacin is also responsible for hyperglycaemia [10,12-14]. other fluoroquinolones as ciprofloxacin, levofloxacin and moxifloxacin have also been associated with hypo- or hyperglycaemic effects which need further attention [12,14-17]. As impact of dysglycemia might not be significant in majority of patients, diabetics are more prone to be affected by this dysglycemic side effect of fluoroquinolones [18-20]. Diabetic patients whether insulin dependent or not are more sensitive to changes in their blood glucose level [18-20]. Thus, any fluctuation in their blood glucose level might lead to deleterious or even lifethreatening effects warranting immediate action [18-20]. Another factor which might precipitate to dysglycemia in diabetic patients is the interaction between fluoroquinolones and oral hypoglycaemic agents especially sulfonyl urea $[17,21]$. Risk factors for dysglyce- 
mia have been identified in various papers including concomitant use of oral hypoglycaemic agents, declined renal functions (renal impairment), body mass index $\leq 30 \mathrm{~kg} / \mathrm{m}^{2}$ and interactions with other drug classes causing hypoglycaemia as beta blockers $[14,17]$.

Despite the main mechanism of dysglycemic effect of fluoroquinolone not well established yet, there are some studies tried to explain it, therefore the mechanism of hypoglycemia may be due to sulphonyl urea-like action, that causes excessive insulin secretion in the first 3 days, then due to pancreatic evacuation of insulin, hyperglycemia may occur in the next two to six days of initiation of quinolones treatment [22]. Another mechanism which may be due to drug-drug interactions through CYP450 isoenzymes or other types of interactions (Ghandour and Azar).

This study is designed to assess the glycaemic changes occurring in diabetic patients during fluoroquinolone treatment and the prevalence of inappropriate prescription of fluoroquinolones in ICU patients. Three fluoroquinolones were included in the study: Ciprofloxacin, Levofloxacin, and moxifloxacin. Demographics and risk factors were identified to detect if significant correlation exist between them and glycaemic changes occurring.

\section{Methods and Materials}

Study design and setting

It is a retrospective observational study targeting the ICU patients who were administered levofloxacin, ciprofloxacin, and moxifloxacin. This study was performed during the period from April 2019 to September 2019 (6 months) in 5 different hospitals in Alexandria, Egypt.

\section{Study population}

A 350 files of ICU patients in the 5 hospitals were randomly checked, then 180 files are excluded as patients' history are not diabetic. After checking for patients' medications from selected files we exclude 78 files that their treatment did not contain fluoroquinolones. To assess the dysglycemic effect of fluoroquinolones we need two random blood sugar level (BSL) readings from hospital Laboratory, to ensure accuracy of results, one at initiation of fluoroquinolone (Baseline BSL) and the second one at day 3-5 from starting quinolone dose (Final BSL), therefore we exclude 34 files. Then a total of 68 diabetic ICU admitted patients receiving broadspectrum fluoroquinolones for more than 2 days were included in the study. Inclusion and exclusion data are represented in flow diagram (Figure 3).

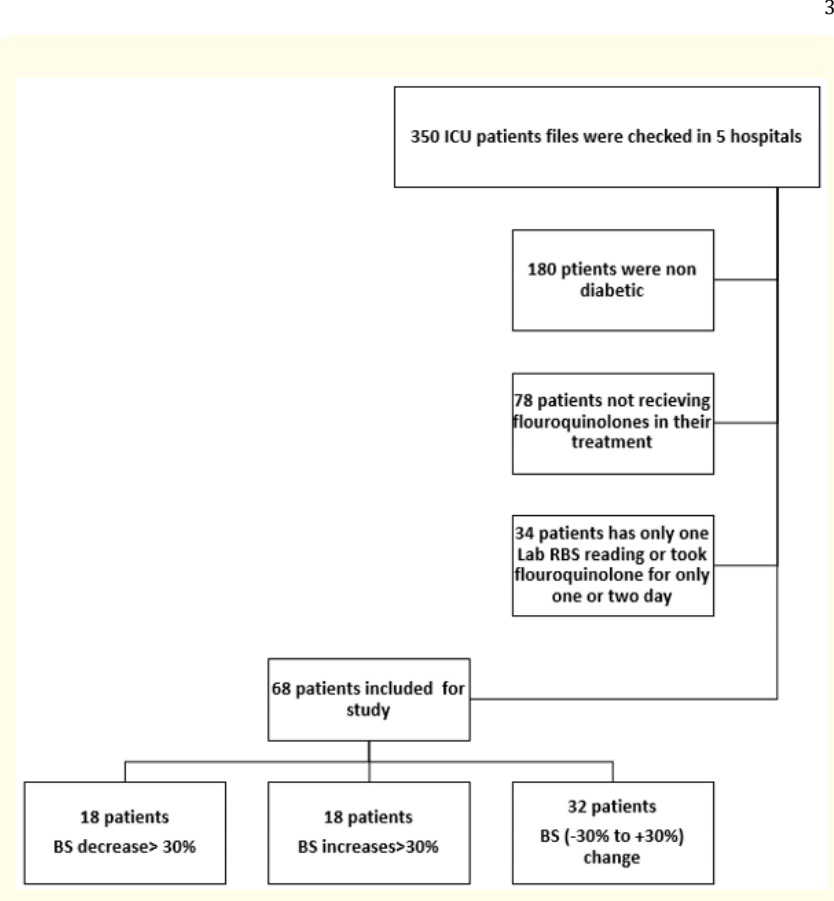

Figure 3: Flow diagram for sample selection. Study antibiotics were moxifloxacin, ciprofloxacin, levofloxacin.

All studied population are patients previously diagnosed as diabetic before ICU admission and they are taken nearly the same nutrition regimens in these ICU units.

Data source and ethics

Data were obtained from Patients' profiles from 5 different hospitals' Databases in Alexandria. Each patient file included the patient's chief complaint, the admission date, past medical history, history of present illness, ICU length of stay, whether the patient is ventilated or not, and all current medications with their doses, and durations of therapy.

Patients' Blood glucose levels were followed up retrospectively from the day the Fluoroquinolone treatment was initiated to the day the patient was discharged from the ICU. Data were obtained after the permission of the responsible crew with regards to ethics and patients' privacy (using the patients' initials and their file number only).

A paper form was prepared to assess the glycaemic changes during the Fluoroquinolone treatment.

The form contained the patients' demographic data, the medical and medication history, the co-administered drugs, the baseline

Citation: Rasha Ashmawy., et al. "Dysglycemic Effect and Misuse Prevalence of Fluoroquinolones in ICU Diabetic Patients". Acta Scientific Pharmaceutical Sciences 4.12 (2020): 36-46. 
BSL, ICU length of stay, renal impairment and the final BSL readings. Any change in the BSL during the treatment was recorded and the medical intervention (if present) in case of dysglycemia was mentioned.

\section{Statistical analysis}

Data were analysed using the Statistical Package for Social Science (SPSS) ver.23. All qualitative variables are presented as counts and percentages, Chi-squared test and Fischer exact test were used to test for statistical significance. Quantitative variables that were not normally distributed and are reported as medians and interquartile range, Mann-Whitney and Kruskal Wallis tests were used to test for statistical significance. Quantitative variables that were normally distributed and are reported as mean and standard deviation, t-tests were used to test for statistical significance.

\section{Results}

Distribution of fluoroquinolone use with patient's diagnosis

Fluoroquinolone treatment was indicated for the resolution of different conditions as in table 1.

Fluoroquinolones used were ciprofloxacin (19.12\%), levofloxacin $(79.41 \%)$ and moxifloxacin $(1.47 \%)$.
Clinical infectious indications included hospital acquired pneumonia (HAP) (17.6\%), Ventilator acquired pneumonia (VAP) (14.7\%), Community Acquired pneumonia (CAP) (14.7\%), sepsis (4.4\%), COPD exacerbation (23.5\%).Other non-infectious indications $(22.1 \%)$, which is a misuse of this important class. That means 15 from 68 patients who recruited in our study take fluoroquinolone with no need and they suffer from change in their blood glucose level without any additional benefit. From table 2 the most misused fluoroquinolone was Levofloxacin, as $73.3 \%$ of patients with main diagnosis non-infectious treated with Levofloxacin, and $20 \%$ among them suffered from decreased blood glucose level where $13.3 \%$ had significant increase of their blood sugar level.

\section{Distribution of variables with dysglycemia}

\section{Qualitative variables}

A number of variables have been assessed against changes in blood glucose levels to detect their effect on dysglycemia in patients using fluoroquinolones (Table 3). Variables were gender, and comorbidities as presence or absence of renal impairment, hypertension, ischemic heart disease, systolic heart failure, ventilatory support, in addition to combined antibiotics used and concomitant drugs which can interfere with blood glucose level.

\begin{tabular}{|c|c|c|c|c|c|}
\hline $\begin{array}{l}\text { Main diagnosis } \\
\text { (Count \& \%) }\end{array}$ & Ciprofloxacin & Levofloxacin & Moxifloxacin & $\begin{array}{l}\% \text { from total } \\
\text { indications }\end{array}$ & P-value \\
\hline VAP & $3(30 \%)$ & $7(70 \%)$ & 0 & $10(14.7 \%)$ & \multirow{6}{*}{0.209} \\
\hline HAP & $1(8.3 \%)$ & $11(91.7 \%)$ & 0 & $12(17.6 \%)$ & \\
\hline CAP & $1(8.3 \%)$ & $10(83.3 \%)$ & $1(83 \%)$ & $12(17.6 \%)$ & \\
\hline Sepsis & $2(66.7 \%)$ & $1(33.3 \%)$ & 0 & $3(4.4 \%)$ & \\
\hline COPD exacerbation & $2(12.5 \%)$ & $14(87.5 \%)$ & 0 & $16(23.5 \%)$ & \\
\hline $\begin{array}{l}\text { Non-infectious indication (Cardiac, DVT, AKI, } \\
\text { Etc.) (Misuse) }\end{array}$ & $4(26.7 \%)$ & $11(73.3 \%)$ & 0 & $15(22.1 \%)$ & \\
\hline Total & 13 & 54 & 1 & $68(100 \%)$ & \\
\hline
\end{tabular}

Table 1: Fluoroquinolones used and patients' main diagnosis.

*VAP: Ventilator Associated Pneumonia; HAP: Hospital Acquired Pneumonia; CAP: Community Acquired Pneumonia; COPD: Chronic Obstructive Pulmonary Disease; DVT: Deep Vein Thrombosis; AKI: Acute Kidney Injury.

\begin{tabular}{|l|c|c|c|c|}
\hline Fluoroquinolones & BSL decrease> 30\% & BSL increases> 30\% & $\begin{array}{c}\text { BSL (-30\% to +30\%) } \\
\text { change }\end{array}$ & Total \\
\hline Ciprofloxacin & $1(6.7 \%)$ & $1(6.7 \%)$ & $2(13.3 \%)$ & $4(26.7 \%)$ \\
\hline Levofloxacin & $3(20 \%)$ & $2(13.3 \%)$ & $6(40 \%)$ & $11(73.3 \%)$ \\
\hline
\end{tabular}

Table 2: Misused quinolones and dysglycemia.

*BSL decrease > 30\%: Blood sugar level (BSL) decreases more than 30\% from initial BSL.

BSL increase > 30\%: Blood sugar level (BSL) increases more than 30\% from initial BSL.

BSL $(-30 \%$ to $+30 \%)$ change: Blood sugar level (BSL) decreases less than $30 \%$ or increases Less than 30 from initial BSL, that couldn't harm patients.

Citation: Rasha Ashmawy., et al. "Dysglycemic Effect and Misuse Prevalence of Fluoroquinolones in ICU Diabetic Patients". Acta Scientific Pharmaceutical Sciences 4.12 (2020): 36-46. 


\begin{tabular}{|c|c|c|c|c|c|}
\hline $\begin{array}{l}\text { Variable } \\
\text { (count \& \%) }\end{array}$ & $\begin{array}{c}\text { BSL decrease> } \\
\mathbf{3 0} \%\end{array}$ & BSL increases $>30 \%$ & $\begin{array}{l}\text { BSL }(-30 \% \text { to } \\
+30 \%) \text { change }\end{array}$ & Total & $\begin{array}{c}\text { P- } \\
\text { value }\end{array}$ \\
\hline \multicolumn{6}{|l|}{ Gender } \\
\hline Male & $9(25 \%)$ & $12(33.3 \%)$ & $15(41.7 \%)$ & 36 & 0.387 \\
\hline Female & $9(28 \%)$ & $6(19 \%)$ & $17(53 \%)$ & 32 & \\
\hline \multicolumn{6}{|c|}{ Renal impairment } \\
\hline No & $11(24.4 \%)$ & $12(26.7 \%)$ & $22(48.9 \%)$ & 45 & 0.859 \\
\hline Yes & $7(30.4 \%)$ & $6(26.1 \%)$ & $10(43.5 \%)$ & 23 & \\
\hline \multicolumn{6}{|c|}{ Ischemic heart disease } \\
\hline No & $16(29.1 \%)$ & $14(25.5 \%)$ & $25(45.5 \%)$ & 55 & 0.721 \\
\hline Yes & $2(15.4 \%)$ & $4(30.8 \%)$ & $7(53.8 \%)$ & 13 & \\
\hline \multicolumn{6}{|c|}{ Systolic heart failure } \\
\hline No & $16(25.8 \%)$ & $16(25.8 \%)$ & $30(48.4 \%)$ & 62 & 0.642 \\
\hline Yes & $2(33.3 \%)$ & $2(33.3 \%)$ & $2(33.3 \%)$ & 6 & \\
\hline Hypertension & & & & & 0.310 \\
\hline No & $14(32.6 \%)$ & $11(25.6 \%)$ & $18(41.9 \%)$ & 43 & \\
\hline Yes & $4(16 \%)$ & $7(28 \%)$ & $14(56 \%)$ & 25 & \\
\hline \multicolumn{6}{|l|}{ Ventilation } \\
\hline No & $13(33.3 \%)$ & $10(25.6 \%)$ & $16(41 \%)$ & 39 & 0.308 \\
\hline Yes & $5(17.2 \%)$ & $8(27.6 \%)$ & $16(55.2 \%)$ & 29 & \\
\hline \multicolumn{6}{|c|}{ Fluoroquinolone } \\
\hline Ciprofloxacin & $2(15.4 \%)$ & $3(23.1 \%)$ & $8(61.5 \%)$ & 13 & 0.432 \\
\hline Levofloxacin & $16(29.6 \%)$ & $14(25.9 \%)$ & $24(44.4 \%)$ & 54 & \\
\hline Moxifloxacin & 0 & $1(100 \%)$ & 0 & 1 & \\
\hline \multicolumn{6}{|c|}{ Combined antibiotics used } \\
\hline \multicolumn{6}{|l|}{ Cephalosporins } \\
\hline None & $9(24.3 \%)$ & $8(21.6 \%)$ & $20(54.1 \%)$ & 37 & 0.585 \\
\hline Cefotaxime & $4(44.4 \%)$ & $2(22.2 \%)$ & $3(33.3 \%)$ & 9 & \\
\hline Ceftriaxone & $2(25.0 \%)$ & $4(50.0 \%)$ & $2(25.0 \%)$ & 8 & \\
\hline Ceftazidime & $3(21.4 \%)$ & $4(28.6 \%)$ & $7(50.0 \%)$ & 14 & \\
\hline \multicolumn{6}{|l|}{ Meropenem } \\
\hline No & $12(24.5 \%)$ & $13(26.5 \%)$ & $24(49 \%)$ & 49 & 0.820 \\
\hline Yes & $6(31.6 \%)$ & $5(26.3 \%)$ & $8(42.1 \%)$ & 19 & \\
\hline \multicolumn{6}{|l|}{ Linezolid } \\
\hline No & $15(24.2 \%)$ & 17 (27.4\%) & $30(48.4 \%)$ & 62 & 0.391 \\
\hline Yes & $3(50 \%)$ & $1(16.7 \%)$ & $2(33.3 \%)$ & 6 & \\
\hline \multicolumn{6}{|l|}{ Amikacin } \\
\hline No & $18(28.1 \%)$ & $17(26.6 \%)$ & $29(45.3 \%)$ & 64 & 0.800 \\
\hline Yes & 0 & $1(25 \%)$ & $3(75 \%)$ & 4 & \\
\hline
\end{tabular}




\begin{tabular}{|l|c|c|c|c|c|}
\hline Other prescribed drugs that could interfere with Blood sugar level \\
\hline B-Blocker & $14(31.1 \%)$ & $10(22.2 \%)$ & $21(46.7 \%)$ & 45 & 0.369 \\
No & $4(17.1 \%)$ & $8(34.8 \%)$ & $11(47.8 \%)$ & 23 & \\
Yes & & & & & \\
\hline ACEIs & $14(25.9 \%)$ & $14(25.9 \%)$ & $26(48.2 \%)$ & 54 & 0.999 \\
No & $4(28.6 \%)$ & $4(28.6 \%)$ & $6(42.8 \%)$ & 14 & \\
Yes & $13(33.3 \%)$ & $9(23.1)$ & $17(43.6 \%)$ & 39 & 0.323 \\
\hline Systemic corticosteroids & $5(17.3 \%)$ & $9(31 \%)$ & $15(51.7 \%)$ & 29 & \\
No & $7(23.3 \%)$ & $7(23.3 \%)$ & $16(53.3 \%)$ & 30 & 0.654 \\
Yes & $11(28.9 \%)$ & $11(28.9 \%)$ & $16(42.1 \%)$ & 38 & \\
\hline Furosemide & & & & & \\
No & $17(26.6 \%)$ & $18(28.1 \%)$ & $29(45.3 \%)$ & 64 & 0.800 \\
Yes & $1(25 \%)$ & 0 & $3(75 \%)$ & 4 & \\
\hline Noradrenalin & & & & & \\
No & & & & & \\
Yes & & & & & \\
\end{tabular}

Table 3: Distribution of qualitative variables with dysglycemia.

Quantitative variables

Quantitative variables including age, ICU days and number of fluoroquinolone treatment days were identified as in table 4 by median and range due to abnormal distribution. Blood glucose changes were identified as more than $30 \%$ increase or decrease from initial BSL levels.

Analytical Results:

Data were analysed using the Statistical Package for Social Science (SPSS) ver.23. Tests performed were Kruskal Wallis test (Table 5 and 6). Results of Kruskal Wallis test with a P-value of 0.003 and $<0.0005$ indicates significant change in initial and final Blood sugar level between dysglycemia classes (Figure 4 and 5) (Table 5). On the other hand (Table 6) and (Figure 6) shows non-significant P-value for Kruskal Wallis test for distribution of blood sugar level percent among Fluoroquinolones used.

Test for correlation was carried out using Spearman's Rho test (Table 7). Results indicate no significant correlation between blood glucose level changes and variables tested (Age, ICU days and fluoroquinolone treatment days) at a level of significance of 0.05 (2-tailed).

\begin{tabular}{|l|c|c|c|}
\hline $\begin{array}{l}\text { Variable } \\
\text { (Median and range) }\end{array}$ & BSL decrease> 30\% & BSL increases $>\mathbf{3 0 \%}$ & $\begin{array}{c}\text { BSL } \mathbf{( - 3 0 \%} \text { to +30\%) } \\
\text { change }\end{array}$ \\
\hline Age & $64.5(37-82)$ years & $61.5(48-85)$ years & $64(42-82)$ years \\
\hline ICU Days & $8(3-19)$ days & $8(4-17)$ days & $8(2-21)$ days \\
\hline $\begin{array}{l}\text { Fluoroquinolone } \\
\text { treatment days }\end{array}$ & $5(3-14)$ days & $6(3-16)$ days & $5(4-14)$ \\
\hline
\end{tabular}

Table 4: Age, ICU days and Fluoroquinolone treatment days.

\begin{tabular}{|l|c|c|c|c|}
\hline $\begin{array}{l}\text { Variable } \\
\text { (Median and range) }\end{array}$ & BSL decrease $>$ 30\% & BSL increases>30\% & $\begin{array}{c}\text { BSL (-30\% to +30\%) } \\
\text { change }\end{array}$ & P-value \\
\hline Initial blood sugar & $245(132-640) \mathrm{mg} / \mathrm{dl}$ & $184(76-336) \mathrm{mg} / \mathrm{dl}$ & $160(74-450) \mathrm{mg} / \mathrm{dl}$ & 0.003 \\
\hline Final blood sugar & $160(74-450) \mathrm{mg} / \mathrm{dl}$ & $342(160-685) \mathrm{mg} / \mathrm{dl}$ & $145(78-425) \mathrm{mg} / \mathrm{dl}$ & $<0.0005$ \\
\hline
\end{tabular}

Table 5: Relation between initial of final blood sugar level and dysglycemia. 


\begin{tabular}{|l|c|c|c|c|}
\hline $\begin{array}{l}\text { Variable } \\
\text { (Median and range) }\end{array}$ & Ciprofloxacin & levofloxacin & Moxifloxacin & P -value \\
\hline BSL percent change & $-5.55 \%(-53 \%$ to $270 \%)$ & $-10 \%(-58 \%$ to $156 \%)$ & $111 \%$ & 0.562 \\
\hline
\end{tabular}

Table 6: Relation between blood sugar level percent change and fluroquinolone type.

\begin{tabular}{|c|c|c|c|c|c|c|c|}
\hline & & & Initial BSL & $\begin{array}{l}\text { Final } \\
\text { BSL }\end{array}$ & Age & ICU days & $\begin{array}{c}\text { Fluoroquinolone } \\
\text { days }\end{array}$ \\
\hline \multirow[t]{3}{*}{ Spearman's rho } & \multirow[t]{3}{*}{ BSL change } & Correlation Coefficient & $-.332^{* *}$ & $.706^{* *}$ & -.007 & -.031 & .005 \\
\hline & & Sig. (2-tailed) & .006 & .000 & .954 & .801 & .967 \\
\hline & & $\mathrm{N}$ & 68 & 68 & 68 & 68 & 68 \\
\hline
\end{tabular}

Table 7: Correlation between quantitative variables with blood sugar level change.

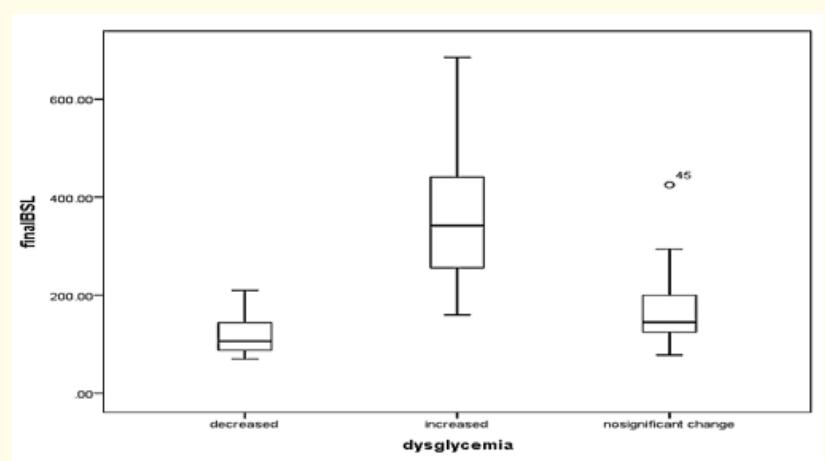

Figure 4: Relation between initial blood sugar level and dysglycemia.

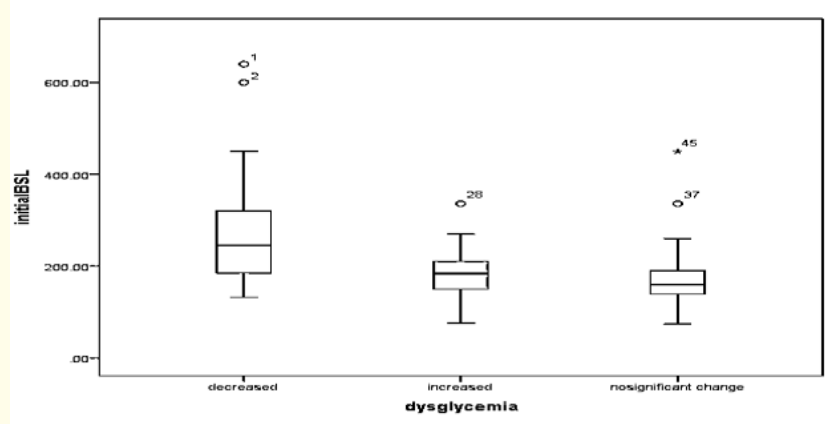

Figure 5: Relation between final blood sugar level and dysglycemia.

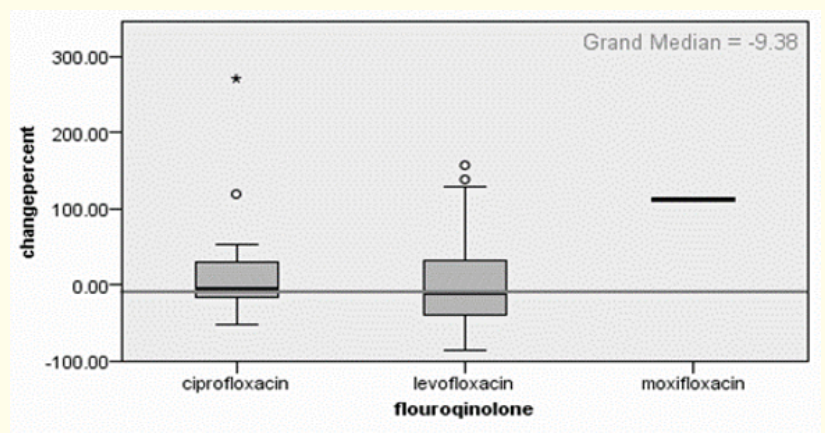

Figure 6: Relation between blood sugar level percent change and fluroquinolone type.

\section{Discussion}

Fluoroquinolones are an important class of antibiotics used in various infectious clinical conditions [1-4]. Apart from their toxicity profile causing QTc prolongation, tendinitis, phototoxicity, renal and liver damage, these antimicrobials offer a great option as treatment of many infections [1-4]. Therefore, their use needs to limit for a suitable indication, and any misuse can harm patients from unwanted adverse effects, in addition to, increase percentages of multidrug resistant organisms. Reports of dysglycemia with quinolone therapy have been increasing in the last years whether hypoor hyperglycaemia [9,11-17]. Disturbances in blood glucose levels is an important aspect that must be taken into consideration when prescribing such antibiotics especially in diabetics or patients with renal impairment [14,17-20]. 
We conducted a study to assess dysglycemic effect of three fluoroquinolones which are ciprofloxacin. levofloxacin and moxifloxacin. Our findings proved that those fluoroquinolones are associated with dysglycemia whether hypo- or hyperglycaemia with varying degrees as shown in figure 7. As shown in the graph, Levofloxacin is more associated with dysglecmia than cipro- and moxifloxacin. Moxifloxacin has the lowest incidence of dysglycemia. Moxifloxacin causes only hyperglycemia. Ciprofloxacin causes both increase and decrease in BSL but nonsgnificant changes is of higher incidence which might reflect that it has no significant effect on blood glucose levels as compared to the other two agents. Correlation between age, ICU stay and days of fluoroquinolones treatment and the incidence of dysglycemia was assessed, with no siginficatnt results.

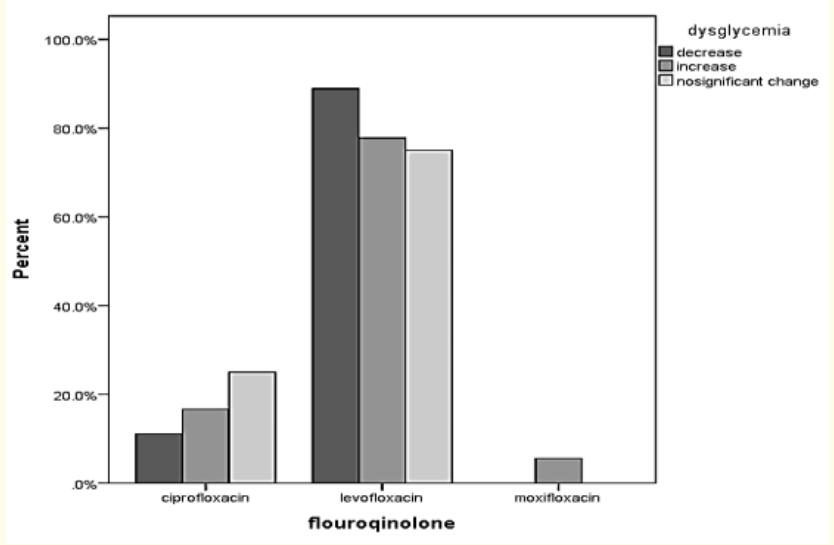

Figure 7: Percentage of BSL change with fluoroquinolones.

To date, the actual mechanism of hypo- and hyperglycemia caused by fluoroquinolone is not quite clear. Studies have investigated the mechanism of hypoglycemia caused by fluoroquinolones. Studies showed that mechanism of hypoglycemia caused by fluoroquinolones is through inhibition of adenosine triphosphate dependant potassium channel in beta-cells of Ilets of Lanagerhans in pancereas $[23,24]$. This in turn will case depolarization of betacells memberane. Depolarization will cause voltage-dependant calcium channels to open leading to calcium influx pancereas $[23,24]$. Calcium cause insulin secretion which in turn leads to derease in blood glucose level (hypoglycemia) [23,24]. These effects seen when fluoroquinolones used on short term. On long term, insulin synthesis decresases and distintegration of insulin secondary to oversecretion during the short term phase takes place [14-17]. Thus, hyperglycemia takes place. This is mechanism for hyperglycemia is still an assumption yet to be proved.
To date, gatifloxacin is the only fluoroquinolone which has a proven dysglycemic effect and has already been warranted label changes [9-13]. As the dysglycemic effect is dose dependant, dose modifications in renal impairment is warranted [14,17]. Reports have been increasing concerning the dysglycemic effects of othr fluoroquinolones as levofloxacin, ciprofloxacin and moxifloxacin [12,14-17]. This lead to the hypothesis that this dysglycemia is a class effect. Yet studies so far can't prove this hypothesis as dysglycemic events vary between different fluoroquinolones [12]. More studies into this aspect are warranted.

Another issue that should be addressed is fluoroquinolones misuse. Fluoroquinolones are being prescribed for non-infectious/ non-bacterial indications which accounts for emerging resistance $[25,26]$. These antibiotics should be reserved for specific organisms which are resistant to other antibiotics. Other ways of misuse include longer than appropriate duration of use, repeated antimicrobial coverage, inadequate coverage, empiric use of fluoroquinolones as in urinary tract infections (asymptomatic bacteriuria) $[25,26]$. All this leads to fluoroquinolone resistance together with unnecessary side effects that reduce the quality of patients' life $[25,26]$. Thus, appropriate prescription and audit of fluoroquinolones is warranted.

\section{Conclusion}

In conclusion, fluoroquinolones are an important class of antibiotics used in the treatment of various ailments. Toxicity profile has been established years ago and well-known. Dysglycemia caused by fluoroquinolones is an important issue of clinical significance and must be addressed and managed. Dysglycemia occurs by varying degrees and differ between different agents of fluoroquinolones. The effect is more pronounced in diabetics and patients with renal impairment which warrant attention and immediate management. Further investigations related to dysglycemia and its mechanism is needed to get a better understanding of this problem.

\section{Study Limitations}

This study targeted diabetic patients, as they are more sensitive to any change in blood sugar level, but further studies are needed to describe fluoroquinolones dysglycemic effect on other populations. Due to limited number of patients that recruited in the study, we could not clearly prove the association between fluoroquinolones use and hypo- or hyperglycaemia.

\section{Conflict of Interest}

None. 
Appendix

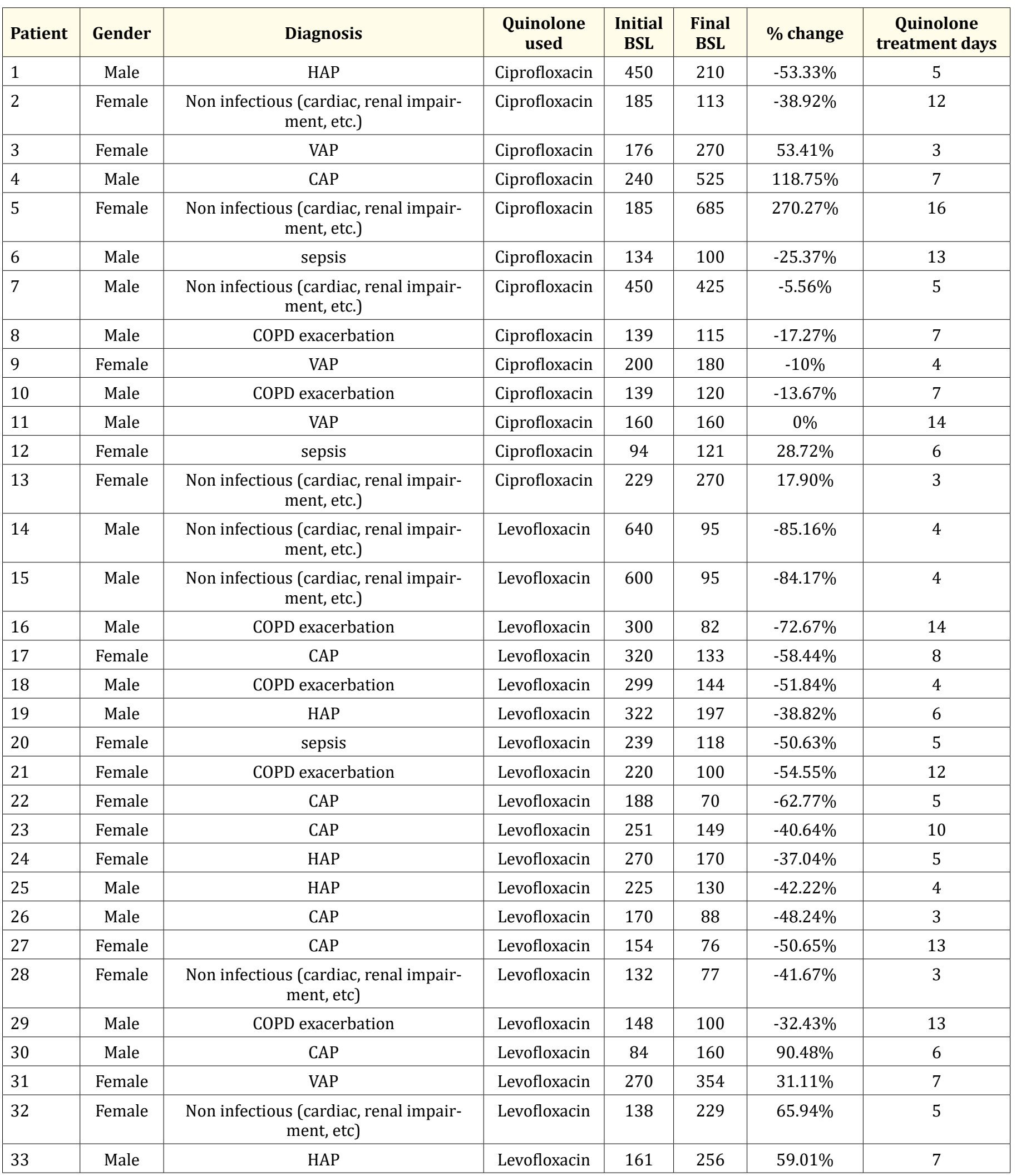

Citation: Rasha Ashmawy., et al. "Dysglycemic Effect and Misuse Prevalence of Fluoroquinolones in ICU Diabetic Patients". Acta Scientific Pharmaceutical Sciences 4.12 (2020): 36-46. 


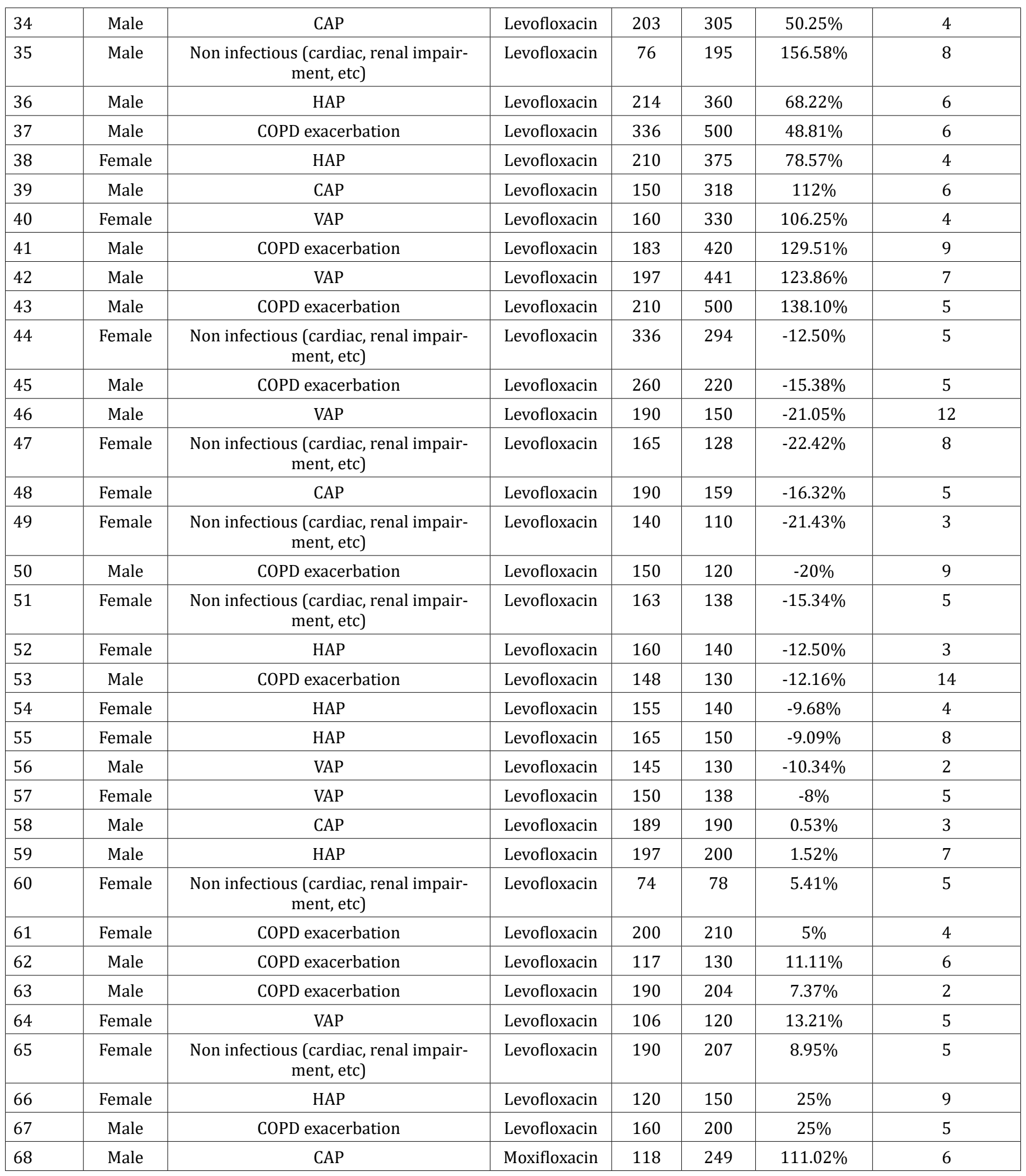




\section{Bibliography}

1. Bambeke F Van., et al. "Quinolones in 2005: An Update". European Society of Clinical Infectious Diseases 11.4 (2005): 256280.

2. Emami Saeed., et al. "Quinolones: Recent Structural and Clinical Developments". Iranian Journal of Pharmaceutical Research (2005): 123-136.

3. Andriole Vincent T. "The Quinolones: Past, Present, and Future". 41.2 (2005): 113-119.

4. A ODonnell Judith and Steven P Gelone. "FLUOROQUINOLONES". Infectious Disease Clinics of North America 14.2 (2000): 489-513.

5. Rubinstein Ethan. History of Quinolones and Their 47.3 (2001): 3-8.

6. Lipsky Benjamin A and Catherine A Baker. "Fluoroquinolone Toxicity Profiles: A Review Focusing on Newer Agent”. Clinical Infection Disease 28.2 (1999): 352-364.

7. Sarro Angela De and Giovambattista De Sarro. "Adverse Reactions to Fluoroquinolones. An Overview on Mechanistic Aspects". Current Medicinal Chemistry (2001): 371-384.

8. Owens, Robert C and D Pharm. "A Tale of Two Toxicities". 25.10 (2005): 1291-1295.

9. ZVONAR ROSEMARY. "Gatifloxacin-Induced Dysglycemia". American Journal of Health-System Pharmacy 63.21 (2006): 2087-2092.

10. Stumpo Carmine., et al. "Outpatient Gatifloxacin Therapy and Dysglycemia in Older Adults". The New England Journal of Medicine 354 (2006): 1352-1361.

11. Hatton Randy C. "An Evaluation of the Effects of Gatifloxacin on Glucose Homeostasis". Pharmacy World and Science 3.5 (2008): 544-549.

12. Aspinall Sherrie L., et al. "Severe Dysglycemia with the Fluoroquinolones: A Class Effect?” 60141 (2009).

13. Ghandour Sarah El and Sami T Azar. "Dysglycemia Associated with Quinolones". Primary Care Diabetes 9.3 (2014): 168-171.

14. Pharm, D., et al. "A Retrospective, Comparative Evaluation of Dysglycemias in Hospitalized Patients Receiving Gatifloxacin, Levofloxacin, Ciprofloxacin, or Ceftriaxone". 25.10 (2005): 1303-1309.

15. Singh Madhurita., et al. "Fatal Hypoglycemia with Levofloxacin Use in an Elderly Patient in the Post-Operative Periods". Langenbecks Archives of Surgery 393.2 (2008): 235-238.
16. Kapoor Rajan., et al. "Life-Threatening Hypoglycemia With Moxifloxacin in a Dialysis Patient". Journal of Clinical Pharmacology 52.2 (2012): 269-271.

17. Laplante Kerry L., et al. "Prevalence of and Risk Factors for Dysglycemia in Patients Receiving Gatifloxacin and Levofloxacin in an Outpatient Setting" (2008).

18. Stolz Randall R., et al. "Effect of Multiple-Dose Gatifloxacin or Ciprofloxacin on Glucose Homeostasis and Insulin Production in Patients with Noninsulin-Dependent Diabetes Mellitus Maintained with Diet and Exercise". Pharmacotherapy 20 (2000): 76-86.

19. Chou Hsu-wen., et al. "Risk of Severe Dysglycemia Among Diabetic Patients Receiving Levo Fl Oxacin, Cipro Fl Oxacin, or Moxi Fl Oxacin in Taiwan". Clinical Infection Disease 57.7 (2013): 971-98.

20. Whiteley M., et al. "Hypoglycaemia in a Diabetic Patient, Associated with Ciprofloxacin Therapy". Practical Diabetes International 10.7 (1993): 50.

21. Parilo Miguel. "Gatifloxacin-Associated Hypoglycemia". (2002): 2-3.

22. Jose J., et al. "Dysglycemia Associated With The Use Of Fluoroquinolones- Focus On Gatifloxacin. Review Article". Wilderness and Environmental Medicine 25.1 (2014): 114.

23. Saraya Atsunori., et al. "Effects of Fluoroquinolones on Insulin Secretion and h -Cell ATP-Sensitive K + Channels". 497 (2004): 111-117.

24. Iguchi, A. “Increase in Insulin”. (1996): 372-76.

25. Seligman B G S., et al. "Critical Steps in Fluoroquinolones and Carbapenems Prescriptions: Results from a Prospective Clinical Audit". International Journal of Clinical Practice 61.1 (2007): 147-152.

26. Werner Nicole L., et al. "Unnecessary Use of Fluoroquinolone Antibiotics in Hospitalized Patients". BMC Infectious Diseases 11 (2011).

\section{Assets from publication with us}

- Prompt Acknowledgement after receiving the article

- Thorough Double blinded peer review

- Rapid Publication

- Issue of Publication Certificate

- High visibility of your Published work

Website: www.actascientific.com/

Submit Article: www.actascientific.com/submission.php Email us: editor@actascientific.com

Contact us: +919182824667 